\title{
The Development of Cortical Multisensory Integration
}

\author{
Mark T. Wallace, ${ }^{1}$ Brian N. Carriere, ${ }^{2}$ Thomas J. Perrault Jr, ${ }^{2}$ J. William Vaughan, ${ }^{2}$ and Barry E. Stein ${ }^{2}$ \\ ${ }^{1}$ Department of Hearing and Speech Sciences, Vanderbilt Kennedy Center for Research on Human Development, Vanderbilt University, Nashville, \\ Tennessee, and ${ }^{2}$ Department of Neurobiology and Anatomy, Wake Forest University School of Medicine, Winston-Salem, North Carolina 27157
}

\begin{abstract}
Although there are many perceptual theories that posit particular maturational profiles in higher-order (i.e., cortical) multisensory regions, our knowledge of multisensory development is primarily derived from studies of a midbrain structure, the superior colliculus. Therefore, the present study examined the maturation of multisensory processes in an area of cat association cortex [i.e., the anterior ectosylvian sulcus (AES)] and found that these processes are rudimentary during early postnatal life and develop only gradually thereafter. The AES comprises separate visual, auditory, and somatosensory regions, along with many multisensory neurons at the intervening borders between them. During early life, sensory responsiveness in AES appears in an orderly sequence. Somatosensory neurons are present at 4 weeks of age and are followed by auditory and multisensory (somatosensory-auditory) neurons. Visual neurons and visually responsive multisensory neurons are first seen at 12 weeks of age. The earliest multisensory neurons are strikingly immature, lacking the ability to synthesize the cross-modal information they receive. With postnatal development, multisensory integrative capacity matures. The delayed maturation of multisensory neurons and multisensory integration in AES suggests that the higher-order processes dependent on these circuits appear comparatively late in ontogeny.
\end{abstract}

Key words: cross-modal; anterior ectosylvian sulcus; cat; maturation; auditory; somatosensory

\section{Introduction}

It is difficult to overestimate the magnitude and novelty of the environmental shift and the new behavioral demands that an animal experiences after birth. In altricial species such as the cat, these potential difficulties are minimized by an attentive mother who takes on much of the responsibility for protecting her offspring and initiating feeding. However, as the neonate matures, it spends more time away from the mother and must begin taking on increasing responsibility in dealing with environmental challenges (Rosenblatt et al., 1969). This gradual transition toward independence almost certainly depends on the brain developing the ability to use sensory information from multiple modalities to initiate adaptive behaviors.

Critical among these developing behavioral capabilities are sensorimotor transformations such as those involving the midbrain superior colliculus (SC). The maturation of these behaviors has been well documented (Stein and Meredith, 1993) and follows a characteristic chronology with somatosensory responses appearing before birth (Stein et al., 1973), facilitating later orientation to the nipple (Larson and Stein, 1984). These are then followed by the appearance of auditory and then visual responsiveness during postnatal life (Stein et al., 1973; Kao et al., 1994). Multisensory neurons follow a similar, albeit delayed, pattern. However, the earliest multisensory neurons are unable to com-

\footnotetext{
Received July 31, 2006; revised Sept. 7, 2006; accepted 0ct. 10, 2006.

This work was supported by National Institutes of Health Grants MH 63861 and NS 36916. We thank Nancy London for her editorial assistance.

Correspondence should be addressed to Dr. Mark T. Wallace, Department of Hearing and Speech Sciences, Vanderbilt Kennedy Center for Research on Human Development, Vanderbilt University, 46521 st Avenue South, Nashville, TN 37232. E-mail: mark.wallace@vanderbilt.edu.

DOI:10.1523/JNEUROSCI.3295-06.2006

Copyright $\odot 2006$ Society for Neuroscience $\quad$ 0270-6474/06/2611844-06\$15.00/0
}

bine the information they receive from different senses to amplify or degrade the physiological salience of environmental events (Wallace et al., 1997). This process appears only gradually during postnatal life and requires the functional maturation of corticotectal inputs, particularly those from the anterior ectosylvian sulcus (AES) (Wallace et al., 1992; Wallace and Stein, 2000) (see also Wallace et al., 1993; Jiang et al., 2001; Stein, 2005).

However, the importance of higher-order structures such as the AES in the development of the multisensory capabilities of the brain is not limited to the critical influences they provide to structures such as the SC but also includes their intrinsic multisensory processes. In the adult, the AES contains many multisensory neurons, and these integrate multisensory information much like SC neurons (Wallace et al., 1992). Undoubtedly, the maturation of multisensory integration at multiple brain levels is essential for the various sophisticated behavioral, perceptual, and emotive processes that coordinate and characterize complex behaviors. However, the time course and developmental antecedents for multisensory maturation in the cerebral cortex are unknown.

One reasonable inference that is in keeping with current concepts is that these cortical processes develop later in life than do those in the midbrain and require a considerable period of postnatal maturation. However, such an expectation is inconsistent with some theories of human development (Gibson, 1969; Bower, 1974; Marks, 1978) that require those multisensory integration processes underlying perception to be functional in newborns. In light of the impact of such theories [and those of opposing theories (for review, see Lewkowicz and Kraebel, 2004; Lickliter and Bahrick, 2004)], the paucity of direct empirical information in any higher-order model system seems surprising. The intent of the present experiments was to use a well charac- 
terized multisensory cortical model system, the cat AES, to begin exploring this issue.

\section{Materials and Methods}

Procedures were performed in compliance with the Guide for the Care and Use of Laboratory Animals (National Institutes of Health publication 91-3207) at Wake Forest University, which is accredited by the American Association for Accreditation of Laboratory Animal Care. Details of surgery, stimulation, and data analyses are similar to those used previously, and additional methodological detail can be obtained from Wallace et al. (1993) and Wallace and Stein (1997).

General surgical procedures. Cats at five developmental stages $(4,8,12$, 16 , and 20 postnatal weeks) were prepared for electrophysiological recordings. The earliest time point (i.e., 4 weeks) was chosen because preliminary experiments had revealed little sensory-evoked activity in AES at earlier ages. At the two earliest ages, the recordings were done using an acute preparation. At the older ages, animals were implanted with a head holder/recording chamber and recorded from using a semichronic paradigm (McHaffie and Stein, 1983).

For surgery, anesthesia was induced with a combination of ketamine hydrochloride (5-25 mg/kg, i.m.) and acepromazine maleate $(0.2-0.4$ $\mathrm{mg} / \mathrm{kg}, \mathrm{i} . \mathrm{m}$.), and surgical anesthesia was maintained with isofluorane $(1.0-4.0 \%)$. For acute procedures, a craniotomy was made over the AES. A head holder was then attached to the skull using orthopedic screws and orthopedic cement to comfortably support the animal during recording without obstructing the face and ears. For semichronic procedures, a head holder/recording chamber was implanted over a craniotomy providing access to the SC. A second recording chamber was implanted over AES. The chambers were sealed, and the animal recovered from anesthesia. Analgesics and antibiotics were administered in consultation with veterinary staff. Recordings typically began 1 week later.

For recordings, anesthesia (ketamine hydrochloride, $10-25 \mathrm{mg} / \mathrm{kg}$, i.m.; acepromazine malleate, $0.2 \mathrm{mg} / \mathrm{kg}$, i.m.) was induced, and the animal was intubated and artificially respired. The saphenous vein was cannulated for the continuous administration of fluids (lactated Ringer's solution, 4-8 cc/h), anesthetics (ketamine hydrochloride, 4-8 $\mathrm{mg} \cdot \mathrm{kg}^{-1} \cdot \mathrm{h}^{-1}$ ), and neuromuscular blocking agent (pancuronium bromide, $0.1 \mathrm{mg} \cdot \mathrm{kg}^{-1} \cdot \mathrm{h}^{-1}$ ). Core body temperature was monitored and maintained with a circulating hot water pad. Continuous vital sign monitoring (i.e., expiratory $\mathrm{CO}_{2}$, heart rate, and blood pressure) was used to ensure an adequate plane of anesthesia. The pupils were dilated ( $1 \%$ atropine sulfate), and contact lenses were applied to prevent corneal drying and to correct for refractive errors. The location of the optic disc was projected via reverse ophthalamoscopy onto a translucent $91-\mathrm{cm}-$ diameter Plexiglas hemisphere.

Recordings. Parylene-insulated tungsten electrodes (impedance, 2-5 $\mathrm{M} \Omega$ at $1 \mathrm{kHz}$ ) were advanced into the AES with a hydraulic microdrive. Single-unit and multiunit neural activity was recorded and amplified, and discharges were routed to an oscilloscope, audio monitor, and computer for subsequent off-line analyses.

Stimulus delivery, receptive field mapping, and latency determination. The electrode was advanced in $20 \mu \mathrm{m}$ steps, and visual, auditory, and somatosensory search stimuli were presented. Visual stimuli consisted of moving and flashed spots and bars of light projected onto the Plexiglas hemisphere or the illumination of a light-emitting diode. If the neuron exhibited a visual response, its receptive field was mapped on the hemisphere using a hand-held pantoscope. Auditory stimuli consisted of broadband (i.e., pink) noise bursts delivered from speakers mounted on a hoop (radius, $30 \mathrm{~cm}$ ) that could be rotated around the animal. Somatosensory stimuli consisted of manual deflections of the hair or skin using a camel's hair brush, blunt probe, or air puff. All receptive fields were transferred onto standard representations of visual, auditory, and somatosensory space (Stein and Meredith, 1993). Mean response latency was determined in response to 10 iterations of a stimulus $2 \times$ threshold intensity.

Multisensory tests and analyses. A multisensory neuron was defined as one that responded to or was influenced by stimuli from more than one sensory modality (Stein and Meredith, 1993).

Once a multisensory neuron was identified, its sensory responses were quantitatively evaluated by randomly interleaving the single-modality and cross-modality stimuli at intervals of $10-15 \mathrm{~s}$. The mean response to the stimulus combination was then statistically compared with the response to the most effective single-modality component stimulus (Student's $t$ test, $p<0.05$ ). Multisensory enhancement was defined as a significant increase in the number of impulses to the combined stimuli compared with the most effective single-modality stimulus. This is considered to be the most robust measure off multisensory integration, because it is evident in virtually all neurons showing this capacity [compared with multisensory depression, which is seen in only a subset of these neurons (Kadunce et al., 1997)]. The magnitude of multisensory response enhancement was calculated as follows: $\left(\mathrm{CM}-\mathrm{SM}_{\max }\right) /$ $\left(\mathrm{SM}_{\max }\right) \times 100=\%$ interaction, where $\mathrm{CM}$ is the mean number of impulses evoked by the combined-modality stimulus, and $\mathrm{SM}_{\max }$ is the mean number of impulses evoked by the most effective single-modality stimulus.

Histological analyses. The depth of each recorded neuron was noted in each electrode penetration, and electrolytic marking lesions were made to facilitate the reconstruction of neuronal locations. At the end of the final recording experiment, the animal was killed with sodium pentobarbital (100 mg/kg, i.v.) and perfused transcardially with saline, followed by Formalin. The midbrain was stereotaxically blocked, removed, and placed in sucrose overnight. Frozen coronal sections (50 $\mu \mathrm{m}$ thickness) were counterstained with neutral red. The outlines of the tissue and laminar boundaries were drawn, and electrode tracts and lesions were traced using a projection microscope. Measurements (after accounting for tissue shrinkage) were entered into a Macintosh PowerPC personal computer (Apple Computers, Cupertino, CA) via a digitizing pad.

To pool data across different animals at a given age, a template AES was created that most closely approximated the sulcal morphology in the largest number of cases at that age. Because penetrations were done while directly viewing the sulcus, this allowed a replotting of the penetrations onto the template AES using standard fiduciary landmarks as references.

\section{Results \\ Sampling}

Although there is substantial development of AES after birth, the sulcus is already well formed and clearly evident before the second week of postnatal life (Jiang et al., 2006). Recording experiments began with animals 4 weeks after birth and at 4 week intervals thereafter, resulting in sampling at $4(n=4), 8(n=4), 12$ $(n=3), 16(n=3)$, and $20(n=2)$ weeks of age, as well as in adults $(n=3)$. The neuronal sampling strategy used electrode penetrations at $1 \mathrm{~mm}$ separations into the dorsal and ventral banks of AES. Because the caudalmost aspect of AES is internalized beneath the cortical mantle, electrode penetrations were extended $3 \mathrm{~mm}$ caudal to the rear end of the visible sulcus.

\section{The appearance and maturation of sensory responses in AES Somatosensory}

At 4 postnatal weeks, the majority (64\%, 37 of 58 ) of isolated AES neurons failed to respond to any of the visual, auditory, and/or somatosensory "search" stimuli. Most had modest spontaneous firing rates (mean, $2.9 \pm 2.7 \mathrm{~Hz}$ ) that, although higher, were not significantly different from those observed in adult animals (mean, $1.8 \pm 1.3 \mathrm{~Hz}$ ). Those neurons that did respond to sensory stimuli $(36 \%, 21$ of 58$)$ were exclusively responsive to somatosensory stimuli (Fig. 1). These early somatosensory neurons were all cutaneous and low threshold, responding best to displacement of the guard hairs [although there was no systematic effort to eliminate the possibility that other hair receptors or even skin receptors were involved (Burgess, 1973)]. Brushing lightly in any direction across the hairs at rapid speeds $(>50 \mathrm{~mm} / \mathrm{s})$ proved to be the most effective stimulus, and no obvious directional preferences were noted.

Compared with their adult counterparts, these early somato- 
sensory neurons had longer response latencies, weaker responses to a suprathreshold stimulus, longer discharge train durations, and more rapid and stronger fatigue to repeated stimulus presentations (Table 1). Perhaps the most distinctive feature of these early somatosensory neurons was their large receptive fields, which typically encompassed the entire body surface (Fig. 2).

Early somatosensory neurons were found in both the dorsal and ventral banks but were restricted to rostral regions in which the AES somatosensory division (SIV) is located (Fig. 1). In contrast, most (30 of 37) unresponsive neurons were located caudal to this somatosensory zone, in the presumptive visual (AEV) and auditory (FAES) subdivisions of the AES.

As detailed in Table 1 and Figure 2, as development progressed, there was a progressive maturation of somatosensory response properties. Response latencies and discharge durations shortened, response strength increased, and receptive fields contracted, until by 20 weeks of age most of these neuronal properties were indistinguishable from those in adults. However, despite a transient rise at 8 weeks, the absolute incidence of somatosensory neurons remained remarkably constant throughout development (Table 2). Of course, the relative proportion of somatosensory neurons decreased as the incidence of other sensory-responsive neuronal types increased (see below).

\section{Auditory}

At 8 weeks of age, the first neurons with auditory responses were isolated in the dorsal bank of the caudal AES. At this age, somatosensory neurons represented $\sim 50 \%$ of the sampled neuronal population, auditory neurons $16 \%$, and unresponsive neurons $34 \%$ (Fig. 1, Table 2). No visually responsive neurons were present at this age. These early auditory AES neurons had immature response properties: their "receptive fields" were typically omnidirectional (Fig. 2), and they had long response latencies, prolonged discharge trains, weaker responses, and were readily fatigued by repeated stimulus presentation (Table 1). Much like with their somatosensory counterparts, there was a gradual maturation of these response characteristics as development progressed. By 12 weeks of age, the incidence of auditory neurons was equivalent to that seen in the adult (Table 2). The spatial distribution of auditory neurons in the developing AES was consistent with the location of the adult FAES, being restricted to the caudal and dorsal aspects (Fig. 1).

\section{Visual}

The first visually responsive neurons were not apparent in AES until 12 weeks after birth. These neurons were primarily distributed in the ventral bank, caudal to the somatosensory representation and ventral to the auditory representation (Fig. 1). As was the case for early auditory and somatosensory neurons, early visual neurons in AES had immature response properties: their receptive fields were very large (Fig. 2), and they had long response latencies, prolonged discharge trains, less vigorous responses, and readily fatigued to repeated stimulus presentations (Table 1). As the animals matured, these response properties became more adult like (Table 1), but, unlike for the somatosensory and auditory populations, some immature properties remained even at the oldest developmental age examined (i.e., 20 weeks). After their appearance, the incidence of visual neurons changes little with age (Table 2).

\section{Multisensory}

The first multisensory neurons were encountered at 8 weeks of age and reflected the two modalities present in AES at this time. These auditory-somatosensory neurons were primarily confined to the dorsal bank of the AES between the major domains of somatosensory and auditory neurons. The first visually responsive multisensory neurons were evident at 12 postnatal weeks, coincident with the age at which the first visual responses were seen. Surprisingly, at this age, visual-responsive multisensory neurons already represented the majority of the multisensory AES population (Fig. 1, Table 2). The incidence of multisensory neurons continued to rise at 16 and 20 postnatal weeks, and, by 20 weeks, they were similar in incidence to the adult. It is interesting to note that several subsets of the multisensory population were of higher incidence at the 20 week time point than in the adult (i.e., visual-somatosensory and visual-somatosensoryauditory neurons). Whether this difference was attributable sim- 
Table 1. Maturation of various unisensory receptive field properties as a function of postnatal age in the AES cortex

\begin{tabular}{|c|c|c|c|c|c|c|}
\hline & 4 weeks & 8 weeks & 12 weeks & 16 weeks & 20 weeks & Adult \\
\hline Somatosensory: latency (ms) ${ }^{\#}$ & $41.8 \pm 27.1^{*}$ & $28.8 \pm 15.4^{*}$ & $23.7 \pm 12.9^{*}$ & $20.0 \pm 9.9$ & $15.6 \pm 8.4$ & $16.2 \pm 6.5$ \\
\hline Somatosensory: discharge duration (ms) & $74.0 \pm 37.4^{*}$ & $33.7 \pm 22.2^{*}$ & $34.5 \pm 20.8^{*}$ & $27.8 \pm 13.8^{*}$ & $20.0 \pm 13.1$ & $17.9 \pm 8.2$ \\
\hline \multicolumn{7}{|l|}{ Somatosensory: response to $2 \times$ threshold } \\
\hline stimulus (spikes/trial) ${ }^{\#}$ & $2.11 \pm 1.47^{*}$ & $2.69 \pm 1.76^{*}$ & $4.27 \pm 1.97$ & $4.02 \pm 2.05$ & $4.93 \pm 2.36$ & $4.70 \pm 1.59$ \\
\hline Auditory: latency (ms) & $28.9 \pm 14.3^{*}$ & $31.3 \pm 14.1^{*}$ & $22.2 \pm 9.8^{*}$ & $18.9 \pm 9.1^{*}$ & $15.7 \pm 6.8$ & $13.7 \pm 5.0$ \\
\hline Auditory: discharge duration (ms) ${ }^{\#}$ & $111.3 \pm 40.0^{*}$ & $72.1 \pm 34.7^{*}$ & $47.7 \pm 23.3^{*}$ & $50.2 \pm 23.6^{*}$ & $42.9 \pm 19.0$ & $35.4 \pm 13.4$ \\
\hline \multicolumn{7}{|l|}{ Auditory: response to $2 \times$ threshold stimulus } \\
\hline (spikes/trial) $^{\#}$ & $1.46 \pm 1.33^{*}$ & $1.82 \pm 1.23^{*}$ & $4.00 \pm 2.38$ & $2.99 \pm 1.78$ & $3.51 \pm 1.66$ & $3.38 \pm 1.50$ \\
\hline Visual: latency (ms) & $154.0 \pm 97.8^{*}$ & $127.3 \pm 69.1^{*}$ & $133.0 \pm 59.8^{*}$ & $114.3 \pm 53.5^{*}$ & $101.8 \pm 45.6^{*}$ & $81.1 \pm 28.7$ \\
\hline Visual: discharge duration (ms) & $208.4 \pm 131.6^{*}$ & $136.3 \pm 78.8^{*}$ & $110.8 \pm 59.0^{*}$ & $99.4 \pm 39.3^{*}$ & $59.8 \pm 33.4^{*}$ & $78.3 \pm 30.7$ \\
\hline \multicolumn{7}{|l|}{ Visual: response to $2 \times$ threshold stimulus } \\
\hline$(\text { spikes/trial) })^{\#}$ & $2.18 \pm 1.60^{*}$ & $4.95 \pm 2.77^{*}$ & $4.5 \pm 2.13^{*}$ & $7.7 \pm 3.43^{*}$ & $8.3 \pm 3.72^{*}$ & $11.7 \pm 4.11$ \\
\hline
\end{tabular}

Mean \pm SD values are shown for each property at each of the sampled ages. "ANOVA revealed a significant main effect of age. ${ }^{*} t$ test revealed significant difference $(p<0.05)$ from adult values.

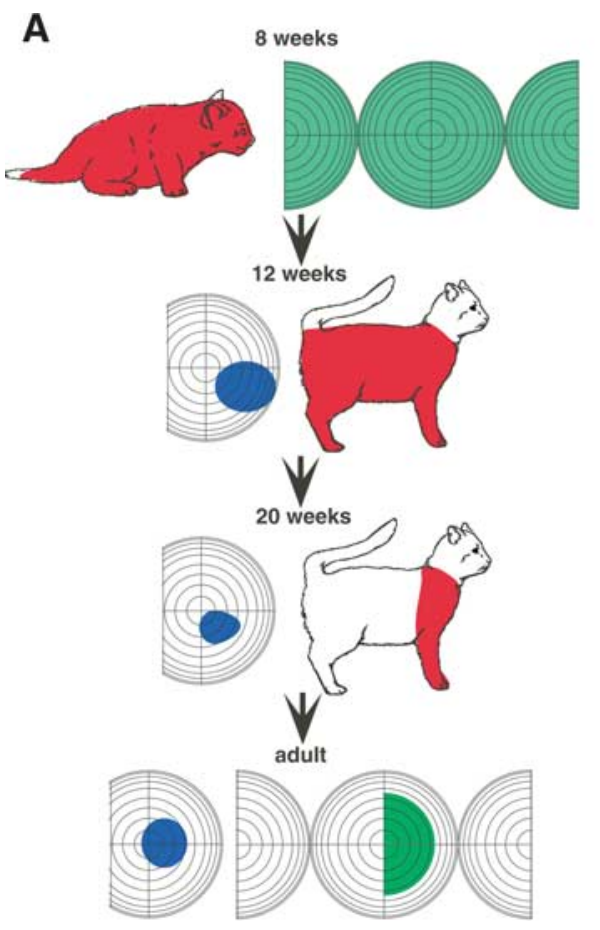

B

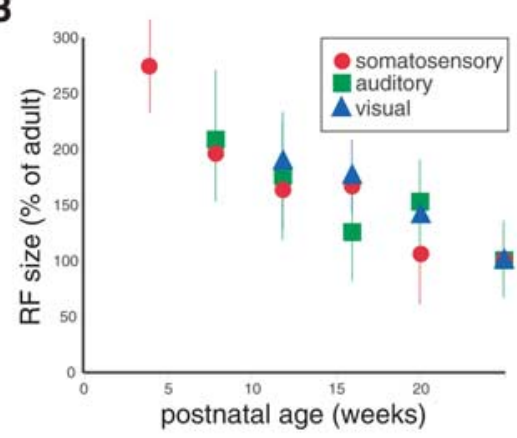

C

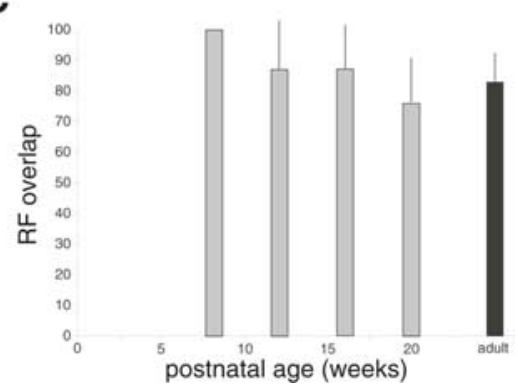

Figure 2. Receptive fields of multisensory AES neurons decline in size as a function of postnatal age. $\boldsymbol{A}$, Example neurons illustrate the shrinkage of receptive fields (RF) (colored shading) and the emergence of good receptive field overlap with maturation. $\boldsymbol{B}$, The decline in mean receptive field size as a function of age. Note that all values are shown as a percentage of adult values. $\boldsymbol{C}$, Average receptive field overlap as a function of age. Error bars in $\boldsymbol{B}$ and $\boldsymbol{C}$ show SDs.

ply to the normal variability in these measures or whether it reflects a true exuberance in these multisensory neuronal types awaits additional investigation.

Early visually responsive multisensory neurons were preferentially located between the major unisensory domains and reflected the modalities represented in these neighboring cortices. The unisensory response properties (e.g., latency, response duration, etc.) of these neonatal multisensory neurons were indistinguishable from their unisensory counterparts at each age. Consequently, the data from both populations have been pooled in the analyses (i.e., Table 1).

The maturation of multisensory integration

Unlike their adult counterparts, early multisensory neurons were unable to integrate their different sensory inputs to enhance their responses. Rather, combined stimulus presentation resulted in responses that were statistically indistinguishable from those to the most effective stimulus component. This is readily evident in the somatosensory-auditory neuron illustrated in Figure $3 A$, which was recorded at the earliest age at which multisensory neurons were found (i.e., 8 weeks). Multisensory integration was not observed in these earliest neurons despite systematic manipulations of the spatial and temporal features of the stimuli, as well as their relative effectiveness. A similar failure to integrate was observed in the earliest visual-nonvisual multisensory neurons.

Later in development, however, neurons exhibited this capacity (Fig. 3B). Paralleling unisensory maturation, the increasing incidence of neurons capable of multisensory integration in AES followed a gradual developmental progression (Fig. $3 C)$. However, the appearance of multisensory integration in any given AES neuron appeared to be quite abrupt, in that the magnitude of the response enhancements evident in the earliest neurons capable of this process was indistinguishable from that found in the adult (Fig. 3D).

\section{Discussion}

The present results reveal a sequential and sensory-specific maturation of AES during postnatal life. The unisensory chronology was one in which the earliest sensoryresponsive neurons ( 4 weeks) were somatosensory, followed by auditory-responsive neurons at 8 weeks and then visually responsive neurons at 12 weeks. This unisensory developmental sequence was predictive of the multisensory chronology, with somatosensory-auditory neurons appearing coincident with the appearance of unisensory auditory neurons and visually responsive multisensory neurons appearing coincident with the appearance of unisensory visual neurons. The appearance and distribution of the different neuronal types in AES was spatially restricted and closely followed the adult functional divisions of AES, with the SIV (Clemo and Stein, 1982, 1983) developing first, the FAES (Clarey and Irvine, 1986) developing second, and the AEV (Mucke et al., 1982; Olson and Graybiel, 1987) developing last.

Adult multisensory AES neurons are preferentially concentrated at the transitions between its unisensory subdivisions (Wallace et al., 1992) (but see Jiang et al., 1994). Such a spatial organization was not violated during development, perhaps be- 
Table 2. Incidence of the different neuronal types in AES as a function of postnatal age

\begin{tabular}{|c|c|c|c|c|c|c|c|c|c|}
\hline Age (weeks postnatal) & Unresp & Som & Aud & Vis & AS & VS & VA & VAS & Total \\
\hline 4 & $0.53(37)$ & $0.30(21)$ & & & & & & & $0.83(58)$ \\
\hline 8 & $0.31(24)$ & $0.47(36)$ & $0.16(12)$ & & $0.04(3)$ & & & & $0.98(75)$ \\
\hline 12 & $0.16(14)$ & $0.27(24)$ & $0.31(28)$ & $0.18(16)$ & $0.02(2)$ & $0.10(9)$ & $0.07(6)$ & $0.02(2)$ & 1.14 (101) \\
\hline 16 & $0.18(12)$ & $0.26(17)$ & $0.31(20)$ & $0.21(13)$ & & $0.12(7)$ & 0.17 (11) & 0.04 (3) & $1.29(83)$ \\
\hline 20 & $0.13(6)$ & $0.26(12)$ & $0.32(15)$ & $0.31(14)$ & & $0.22(10)$ & $0.12(6)$ & $0.09(4)$ & $1.47(67)$ \\
\hline Adult & $0.07(12)$ & $0.33(57)$ & $0.32(55)$ & $0.25(43)$ & $0.03(5)$ & $0.16(29)$ & $0.16(28)$ & $0.05(10)$ & $1.37(239)$ \\
\hline
\end{tabular}

Numbers show the incidence of neurons per millimeter of electrode traverse. Numbers in parentheses show the $n$ for each category. Unresp, Sensory unresponsive; Som, Somatosensory; Aud, auditory; Vis, visual; AS, auditorysomatosensory; VS, visual-somatosensory; VA, visual-auditory; VAS, visual-auditory-somatosensory.

cause this fundamental feature of cortical organization (Barth et al., 1993; Wallace et al., 2004a) (but see Ghazanfar and Schroeder, 2006) is established even before the networks are responsive to sensory stimuli.

The sensory chronology of AES appears to follow a pattern closely paralleling that found in its major subcortical target structure, the SC (Stein et al., 1973). This midbrain structure plays a central role in the transformation of sensory signals into premotor commands (Sparks, 1986; Stein and Meredith, 1993) and depends on AES for its ability to integrate cross-modal inputs to amplify its sensory responses and the behaviors that depend on them (Stein et al., 1989; Wallace et al., 1993; Wilkinson et al., 1996; Wallace and Stein, 2000; Jiang et al., 2001, 2002, 2006). Finally, much like the SC (Wallace and Stein, 1997), the appearance of the amplified multisensory integrative product in any given AES neuron appeared to be an all-or-none event. Once present, and regardless of age, it was indistinguishable from the adult. These parallel sensory chronologies are likely to be independent of interconnections between these regions, because the sensory chronology in the SC is already well established before the functional maturation of AES-SC influences (Wallace and Stein, 2000). Rather, they are likely to reflect a general chronology in sensory development (Gottlieb, 1971). Nonetheless, it remains possible that there is a dynamic developmental interplay between the SC and the AES that is important in shaping the maturation of each of these (multi)sensory representations. Anatomical evidence suggests that AES-SC connectivity is undergoing substantial changes in early postnatal life and may form the substrate for these interactions (McHaffie et al., 1988).

However, the maturational delay between AES and SC conforms to that interval when AES inputs begin influencing the multisensory integrative capabilities of SC neurons. Thus, the AES appears to coordinate the maturation of multisensory capabilities in its intrinsic neurons, presumably involved in higherorder functions, with those of its target neurons in the SC through which it controls orientation behaviors (Wallace and Stein, 2000; Jiang et al., 2006). Furthermore, it appears to exercise this coordination late in the period during which cross-modal experiences critical for multisensory integration are acquired (Wallace et al., 2004b; Wallace and Stein, 2006).

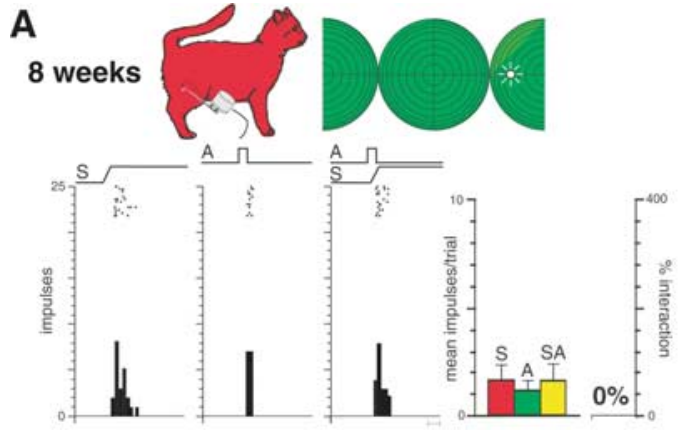

C

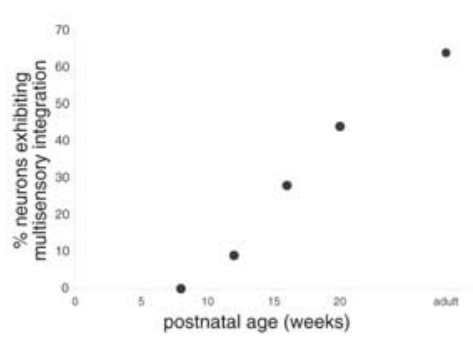

D

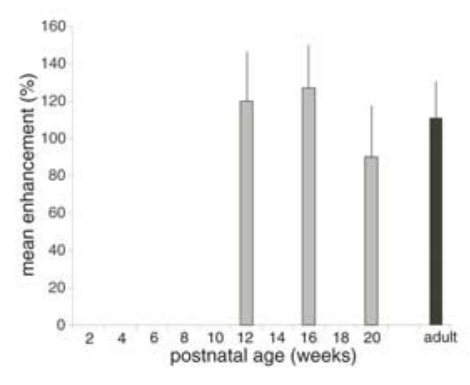

Figure 3. Multisensory integration is absent in the earliest AES neurons and appears and matures during postnatal life. $\boldsymbol{A}$, somatosensory $(S)$, auditory $(A)$, and combined $(S A)$ stimulation. Bar graph at the right summarizes these responses and shows

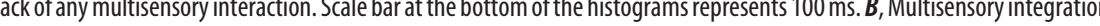
Conventions are the same as in $\boldsymbol{A} .{ }^{*} p<0.01, t$ test. $\boldsymbol{C}$, Growth in the integrating multisensory population as a function of age. $\boldsymbol{D}$, As soon as it appears (i.e., at 12 weeks), multisensory integration is of similar magnitude to the adult. Error bars show the SDs.

Outside of its role in gating subcortical multisensory processing, the behavioral and perceptual functions mediated by AES remain elusive. Although there is some evidence suggesting that AES may be important for the perception of visual stimulus motion (Scannell et al., 1996; Nagy et al., 2003) and sound source localization (Middlebrooks et al., 1998; Furukawa et al., 2000), more work is needed to determine its behavioral and/or perceptual roles.

It is interesting to note that the present data are inconsistent with those theories of multisensory integration that require a newborn nervous system already endowed with functional connections between sensory representations and capable of multisensory integration (Gibson, 1969; Bower, 1974; Marks, 1978). They are more consistent with those positing a gradual and successive recruitment of sensory functions at different levels of the neuraxis (Lewkowicz and Kraebel, 2004; Lickliter and Bahrick, 2004) and the gradual acquisition of intersensory capabilities that depend on the integration of cross-modal cues (Piaget, 1952; Birch and Lefford, 1963). 


\section{References}

Barth DS, Kithas J, Di S (1993) Anatomic organization of evoked potentials in rat parietotemporal cortex: somatosensory and auditory responses. J Neurophysiol 69:1837-1849.

Birch H, Lefford A (1963) Intersensory development in children. Monogr Soc Res Child Dev 28:1-47.

Bower TGR (1974) Development in infancy. San Francisco: Freeman.

Burgess PR (1973) Cutaneous mechanoreceptors. In: Handbook of perception (Carterette EC, Friedman MP, eds), pp 219-249. New York: Academic.

Clarey JC, Irvine DR (1986) Auditory response properties of neurons in the anterior ectosylvian sulcus of the cat. Brain Res 386:12-19.

Clemo HR, Stein BE (1982) Somatosensory cortex: a "new" somatotopic representation. Brain Res 235:162-168.

Clemo HR, Stein BE (1983) Organization of a fourth somatosensory area of cortex in cat. J Neurophysiol 50:910-925.

Furukawa S, Xu L, Middlebrooks JC (2000) Coding of sound-source location by ensembles of cortical neurons. J Neurosci 20:1216-1228.

Ghazanfar AA, Schroeder CE (2006) Is neocortex essentially multisensory? Trends Cogn Sci 10:278-285.

Gibson EJ (1969) Principles of perceptual learning and development. Englewood Cliffs, NJ: Prentice Hall.

Gottlieb G (1971) Ontogenesis of sensory function in birds and mammals. In: The biopsychology of development (Tobach E, Aronson LR, Shaw E, eds), pp 67-128. New York: Academic.

Jiang H, Lepore F, Ptito M, Guillemot JP (1994) Sensory modality distribution in the anterior ectosylvian cortex (AEC) of cats. Exp Brain Res 97:404-414.

Jiang W, Wallace MT, Jiang H, Vaughan JW, Stein BE (2001) Two cortical areas mediate multisensory integration in superior colliculus neurons. J Neurophysiol 85:506-522.

Jiang W, Jiang H, Stein BE (2002) Two corticotectal areas facilitate multisensory orientation behavior. J Cogn Neurosci 14:1240-1255.

Jiang W, Jiang H, Stein BE (2006) Neonatal cortical ablation disrupts multisensory development in superior colliculus. J Neurophysiol 95:1380-1396.

Kadunce DC, Vaughan JW, Wallace MT, Benedek G, Stein BE (1997) Mechanisms of within- and cross-modality suppression in the superior colliculus. J Neurophysiol 78:2834-2847.

Kao CQ, McHaffie JG, Meredith MA, Stein BE (1994) Functional development of a central visual map in cat. J Neurophysiol 72:266-272.

Larson MA, Stein BE (1984) The use of tactile and olfactory cues in neonatal orientation and localization of the nipple. Dev Psychobiol 17:423-436.

Lewkowicz DJ, Kraebel KS (2004) The value of multisensory redundancy in the development of intersensory perception. In: The handbook of multisensory processes (Calvert GA, Spence C, Stein BE, eds), pp 655-678. Cambridge, MA: MIT.

Lickliter R, Bahrick LE (2004) Perceptual development and the origins of multisensory responsiveness. In: The handbook of multisensory processes (Calvert GA, Spence C, Stein BE, eds), pp 643-654. Cambridge, MA: MIT

Marks LE (1978) The unity of the senses: interrelations among the modalities. New York: Academic.

McHaffie JG, Stein BE (1983) A chronic headholder minimizing facial obstructions. Brain Res Bull 10:859-860.

McHaffie JG, Kruger L, Clemo HR, Stein BE (1988) Corticothalamic and corticotectal somatosensory projections from the anterior ectosylvian sulcus (SIV cortex) in neonatal cats: an anatomical demonstration with HRP and 3H-leucine. J Comp Neurol 274:115-126.

Middlebrooks JC, Xu L, Eddins AC, Green DM (1998) Codes for soundsource location in nontonotopic auditory cortex. J Neurophysiol 80:863-881.

Mucke L, Norita M, Benedek G, Creutzfeldt O (1982) Physiologic and anatomic investigation of a visual cortical area situated in the ventral bank of the anterior ectosylvian sulcus of the cat. Exp Brain Res 46:1-11.

Nagy A, Eordegh G, Benedek G (2003) Spatial and temporal visual properties of single neurons in the feline anterior ectosylvian visual area. Exp Brain Res 151:108-114.

Olson CR, Graybiel AM (1987) Ectosylvian visual area of the cat: location, retinotopic organization, and connections. J Comp Neurol 261:277-294.

Piaget J (1952) The origins of intelligence in children. New York: International UP.

Rosenblatt JS, Turkewitz G, Schneirla TC (1969) Development of home orientation in newly born kittens. Trans NY Acad Sci 31:231-250.

Scannell JW, Sengpiel F, Tovee MJ, Benson PJ, Blakemore C, Young MP (1996) Visual motion processing in the anterior ectosylvian sulcus of the cat. J Neurophysiol 76:895-907.

Sparks DL (1986) Translation of sensory signals into commands for control of saccadic eye movements: role of primate superior colliculus. Physiol Rev 66:118-171.

Stein BE (2005) The development of a dialogue between cortex and midbrain to integrate multisensory information. Exp Brain Res 166:305-315.

Stein BE, Meredith MA (1993) The merging of the senses. Cambridge, MA: MIT.

Stein BE, Labos E, Kruger L (1973) Sequence of changes in properties of neurons of superior colliculus of the kitten during maturation. J Neurophysiol 36:667-679.

Stein BE, Meredith M, Huneycutt W, McDade L (1989) Behavioral indices of multisensory integration: orientation to visual cues is affected by auditory stimuli. J Cogn Neurosci 1:12-24.

Wallace MT, Stein BE (1997) Development of multisensory neurons and multisensory integration in cat superior colliculus. J Neurosci 17:2429-2444.

Wallace MT, Stein BE (2000) Onset of cross-modal synthesis in the neonatal superior colliculus is gated by the development of cortical influences. J Neurophysiol 83:3578-3582.

Wallace MT, Stein BE (2006) Early experience determines how the senses will interact. J Neurophysiol, in press.

Wallace MT, Meredith MA, Stein BE (1992) Integration of multiple sensory modalities in cat cortex. Exp Brain Res 91:484-488.

Wallace MT, Meredith MA, Stein BE (1993) Converging influences from visual, auditory, and somatosensory cortices onto output neurons of the superior colliculus. J Neurophysiol 69:1797-1809.

Wallace MT, Ramachandran R, Stein BE (2004a) A revised view of sensory cortical parcellation. Proc Natl Acad Sci USA 101:2167-2172.

Wallace MT, Perrault Jr TJ, Hairston WD, Stein BE (2004b) Visual experience is necessary for the development of multisensory integration. J Neurosci 24:9580-9584.

Wilkinson LK, Meredith MA, Stein BE (1996) The role of anterior ectosylvian cortex in cross-modality orientation and approach behavior. Exp Brain Res 112:1-10. 